\title{
MOMENT LYAPUNOV EXPONENTS AND STOCHASTIC STABILITY FOR TWO COUPLED OSCILLATORS
}

\author{
Predrag Kozić, Goran Janevski and Ratko Pavlović
}

\begin{abstract}
The Lyapunov exponent and moment Lyapunov exponent of two degree-of-freedom linear systems subjected to white noise parametric excitation are investigated. Through a perturbation method we obtain the explicit asymptotic expressions for these exponents in the presence of low intensity noise. The Lyapunov exponent and moment Lyapunov exponents are important characteristics for determining the almostsure and moment stability of a stochastic dynamical system. As an example, we study the almost-sure and moment stability of the flexural-torsion stability of a thin elastic beam subjected to a stochastically fluctuating follower force. The validity of the approximate results for moment Lyapunov exponents is checked by a numerical Monte Carlo simulation for these stochastic systems.
\end{abstract}

\section{Introduction}

In recent years there has been considerable interest in the study of the dynamic stability of nongyroscopic conservative elastic systems whose parameters fluctuate in a stochastic manner. To have a complete picture of the dynamic stability of a dynamic system, it is important to study both the almost-sure and the moment stability and to determine both the maximal Lyapunov exponent and the $p$-th moment Lyapunov exponent. The maximal Lyapunov exponent, defined by

$$
\lambda_{q}=\lim _{t \rightarrow \infty} \frac{1}{t} \log \left\|\boldsymbol{q}\left(t ; q_{0}\right)\right\|,
$$

where $\boldsymbol{q}\left(t ; q_{0}\right)$ is the solution process of a linear dynamical system. The almost-sure stability depends upon the sign of the maximal Lyapunov exponent, which is the exponential growth rate of the solution of the randomly perturbed dynamical system. A negative sign of the maximal Lyapunov exponent implies almost-sure stability, whereas a nonnegative value indicates instability. The exponential growth rate $E\left[\left\|\boldsymbol{q}\left(t ; q_{0}, \dot{q}_{0}\right)\right\|^{p}\right]$ is provided by the moment Lyapunov exponent, defined as

$$
\Lambda_{q}(p)=\lim _{t \rightarrow \infty} \frac{1}{t} \log E\left[\left\|\boldsymbol{q}\left(t ; q_{0}\right)\right\|^{p}\right],
$$

where $E[]$ denotes an expectation. If $\Lambda_{q}(p)<0$, then, by definition $E\left[\left\|\boldsymbol{q}\left(t ; q_{0}, \dot{q}_{0}\right)\right\|^{p}\right] \rightarrow 0$ as $t \rightarrow \infty$. This is referred to as $p$-th moment stability. Although moment Lyapunov exponents are important in the study of the dynamic stability of stochastic systems, the actual evaluation of moment Lyapunov exponents is very difficult.

Arnold et al. [1997] constructed an approximation for the moment Lyapunov exponents of 2D linear systems driven by real or white noise. A perturbation approach was used to obtain explicit expressions for

Keywords: elastic beam, eigenvalue, perturbation, stochastic stability, mechanics of solids and structures.

Research supported by the Ministry of Science and Environmental Protection of the Republic of Serbia, grant No. 144023. 
these exponents in the presence of low intensity noise. Khasminskii and Moshchuk [1998] obtained an asymptotic expansion of the moment Lyapunov exponents of a 2D system under white noise parametric excitation in terms of the small fluctuation parameter $\varepsilon$, from which the stability index was obtained. Kozić et al. [2008] investigated the Lyapunov exponent and moment Lyapunov exponent of Hill's equation with frequency and damping coefficient fluctuated by white noise. A perturbation approach was used to obtain explicit expressions for these exponents in the presence of low intensity noise. Xie [2001] obtained weak noise expansions of the moment Lyapunov exponents of a 2D system under real noise excitation, an Ornstein-Uhlenbeck process. Xie [2002] determined small noise expansions of the moment Lyapunov exponents of a 2D viscoelastic system under bounded noise excitation. Sri Namachchivaya et al. [1994] used a perturbation approach to calculate the asymptotic growth rate of stochastically coupled 4D systems. The noise was assumed to be white and of low intensity in order to calculate the explicit asymptotic formulas for the maximum Lyapunov exponent. Sri Namachchivaya and Vedula [2000] obtained a general asymptotic approximation for the moment Lyapunov exponents and the Lyapunov exponent for 4D systems with one critical mode and another asymptotically stable mode driven by a small intensity process. Sri Namachchivaya and Van Roessel [2004] used a perturbation approach to obtain an approximation for the moment Lyapunov exponents of two coupled oscillators with commensurable frequencies driven by low intensity real noise with dissipation. The generator for the eigenvalue problem associated with the moment Lyapunov exponents was derived without any restrictions on the size of the $p$-th moment.

The aim of this paper is to determine a weak noise expansion for the moment Lyapunov exponents for stochastically coupled two-degree-of-freedom systems. The noise is assumed to be white noise of low intensity such that one can obtain an asymptotic growth rate. Here we apply the perturbation theoretic approach of [Khasminskii and Moshchuk 1998] to obtain weak noise expansions of the moment Lyapunov exponents and Lyapunov exponent. These results are applied to study the flexural-torsional stability of a narrow simply supported beam under a fluctuating stochastic follower force. The approximate analytical results of the moment Lyapunov exponents are compared with the numerical values obtained by Monte Carlo simulation for these exponents of two-degree-of freedom stochastic systems.

\section{Theoretical formulation}

Consider the linear oscillatory systems described by equations of motion of the form

$$
\ddot{q}_{i}+\omega_{i}^{2} q_{i}+2 \varepsilon \zeta_{i} \omega_{i} \dot{q}_{i}+\varepsilon^{1 / 2} \sum_{j=1}^{2} k_{i j} q_{j} \xi(t)=0, \quad i, j=1,2,
$$

where $q_{i}$ is a generalized coordinate, $\omega_{i}$ is the $i$-th natural frequency, and $\varepsilon \zeta_{i}$ represents the $i$-th small viscous damping coefficient. It is assumed that the natural frequency is not commensurable. The stochastic term, $\varepsilon^{1 / 2} \xi(t)$, is a white-noise process with low intensity. The almost-sure and moment stability of the equilibrium state $q=\dot{q}=0$ of (3) is to be investigated. Using the transformation

$$
q_{1}=x_{1}, \quad \dot{q}_{1}=\omega_{1} x_{2}, \quad q_{2}=x_{3}, \quad \dot{q}_{2}=\omega_{2} x_{4},
$$

we can represent (3) in first-order form by the Stratonovich differential equations (4) on the next page which is also the form of the Itô equations: 


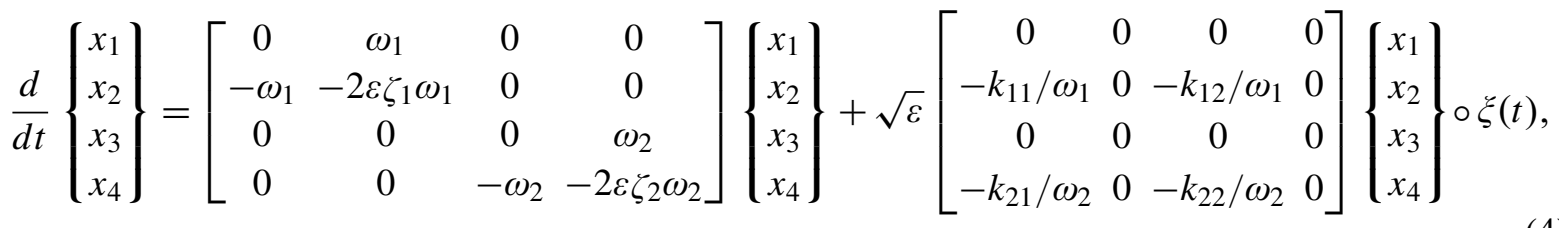

where $\xi(t)$ is the white noise process with zero mean and autocorrelation function

$$
R_{\xi \xi}\left(t_{1}, t_{2}\right)=E\left[\xi\left(t_{1}\right) \xi\left(t_{2}\right)\right]=\sigma^{2} \min \left(t_{2}, t_{1}\right) .
$$

Here $\sigma$ is the intensity of the random process $\xi(t)$ and $\delta$ is the Dirac delta function.

We next apply the transformation

$$
x_{1}=a \cos \phi_{1} \cos \theta, \quad x_{2}=-a \sin \phi_{1} \cos \theta, \quad x_{3}=a \cos \phi_{2} \sin \theta, \quad x_{4}=-a \sin \phi_{2} \sin \theta,
$$

where $\phi_{1}$ and $\phi_{2}$ the angles of the first and second oscillators, and $\theta$ the coupling or energy exchange between the two oscillators. Introducing also

$$
P=a^{p}=\left(x_{1}^{2}+x_{2}^{2}+x_{3}^{2}+x_{4}^{2}\right)^{p / 2}, \quad-\infty<p<\infty,
$$

the $p$-th power of the norm of the response, we obtain a set of Itô equations for $P$ the and phase variables $\phi_{1}, \phi_{2}, \theta$ (where we omit from the notation the dependence of the $m_{i}$ and $\sigma_{i 1}$ on $\phi_{1}, \phi_{2}, \theta$ ):

$$
\begin{array}{ll}
d \phi_{1}=m_{1} d t+\sigma_{11} d W(t), & d \theta=m_{3} d t+\sigma_{31} d W(t), \\
d \phi_{2}=m_{2} d t+\sigma_{21} d W(t), & d P=P m_{4} d t+P \sigma_{41} d W(t) .
\end{array}
$$

Here

$\sigma_{11}=\frac{\sigma}{\omega_{1}} \sqrt{\frac{\varepsilon}{2}}\left(k_{11} \cos \phi_{1}+k_{12} \cos \phi_{2} \tan \theta\right) \cos \phi_{1}, \quad \sigma_{21}=\frac{\sigma}{\omega_{2}} \sqrt{\frac{\varepsilon}{2}}\left(k_{21} \cos \phi_{1} \cot \theta+k_{22} \cos \phi_{2}\right) \cos \phi_{2}$, $\sigma_{31}=\frac{\sigma}{4} \sqrt{\frac{\varepsilon}{2}}\left[\left(\frac{k_{22}}{\omega_{2}} \sin 2 \phi_{2}-\frac{k_{11}}{\omega_{1}} \sin 2 \phi_{1}\right) \sin 2 \theta+4 \frac{k_{21}}{\omega_{2}} \sin \phi_{2} \cos \phi_{1} \cos ^{2} \theta-4 \frac{k_{12}}{\omega_{1}} \sin \phi_{1} \cos \phi_{2} \sin ^{2} \theta\right]$, $\sigma_{41}=\frac{p \sigma}{2} \sqrt{\frac{\varepsilon}{2}}\left[\left(\frac{k_{12}}{\omega_{1}} \sin \phi_{1} \cos \phi_{2}+\frac{k_{21}}{\omega_{2}} \sin \phi_{2} \cos \phi_{1}\right) \sin 2 \theta+\frac{k_{11}}{\omega_{1}} \sin 2 \phi_{1} \cos ^{2} \theta+\frac{k_{22}}{\omega_{2}} \sin 2 \phi_{2} \sin ^{2} \theta\right]$, $m_{1}=\omega_{1}-\varepsilon\left(\zeta_{1} \omega_{1}+\frac{\sigma^{2}}{4 \omega_{1}^{2}}\left(k_{11} \cos \phi_{1}+k_{12} \cos \phi_{2} \tan \theta\right)^{2}\right) \sin 2 \phi_{1}$, $m_{2}=\omega_{2}-\varepsilon\left(\zeta_{1} \omega_{2}+\frac{\sigma^{2}}{4 \omega_{2}^{2}}\left(k_{21} \cos \phi_{1} \cot \theta+k_{22} \cos \phi_{2}\right)^{2}\right) \sin 2 \phi_{2}$, $m_{3}=\frac{\varepsilon}{2}\left(\zeta_{1} \omega_{1}\left(1-\cos 2 \phi_{1}\right)-\zeta_{2} \omega_{2}\left(1-\cos 2 \phi_{2}\right)\right) \sin 2 \theta$ $+\frac{\varepsilon \sigma^{2}}{4}\left[\frac{-\cos ^{2} \phi_{1} \tan \theta+\sin ^{2} \phi_{1} \sin 2 \theta}{\omega_{1}^{2}}\left(k_{11} \cos \phi_{1} \cos \theta+k_{12} \cos \phi_{2} \sin \theta\right)^{2}\right.$ $-\frac{2 \sin \phi_{1} \sin \phi_{2} \cos 2 \theta}{\omega_{1} \omega_{2}}\left(k_{11} \cos \phi_{1} \cos \theta+k_{12} \cos \phi_{2} \sin \theta\right)\left(k_{21} \cos \phi_{1} \cos \theta+k_{22} \cos \phi_{2} \sin \theta\right)$ $\left.+\frac{\cos ^{2} \phi_{2} \cot \theta-\sin ^{2} \phi_{2} \sin 2 \theta}{\omega_{2}^{2}}\left(k_{21} \cos \phi_{1} \cos \theta+k_{22} \cos \phi_{2} \sin \theta\right)^{2}\right]$, 


$$
\begin{aligned}
& m_{4}=-\varepsilon p\left(\zeta_{1} \omega_{1}\left(1-\cos 2 \phi_{1}\right) \cos ^{2} \theta+\zeta_{2} \omega_{2}\left(1-\cos 2 \phi_{2}\right) \sin ^{2} \theta\right) \\
&+\frac{\varepsilon p \sigma^{2}}{4}\left[\frac{1+(p-2) \sin ^{2} \phi_{1} \cos ^{2} \theta}{\omega_{1}^{2}}\left(k_{11} \cos \phi_{1} \cos \theta+k_{12} \cos \phi_{2} \sin \theta\right)^{2}\right. \\
&+\frac{(p-2) \sin \phi_{1} \sin \phi_{2} \sin 2 \theta}{\omega_{1} \omega_{2}}\left(k_{11} \cos \phi_{1} \cos \theta+k_{12} \cos \phi_{2} \sin \theta\right)\left(k_{21} \cos \phi_{1} \cos \theta+k_{22} \cos \phi_{2} \sin \theta\right) \\
&\left.\quad+\frac{1+(p-2) \sin ^{2} \phi_{2} \sin ^{2} \theta}{\omega_{2}^{2}}\left(k_{21} \cos \phi_{1} \cos \theta+k_{22} \cos \phi_{2} \sin \theta\right)^{2}\right],
\end{aligned}
$$

Following [Wedig 1988], we perform the stochastic linear transformation

$$
S=T\left(\phi_{1}, \phi_{2}, \theta\right) P, \quad P=T^{-1}\left(\phi_{1}, \phi_{2}, \theta\right) S .
$$

We introduce the new norm process $S$ by means of the scalar function $T\left(\phi_{1}, \phi_{2}, \theta\right)$ which is defined on the stationary phase processes $\phi_{1}, \phi_{2}$, and $\theta$ in the range $0 \leq \phi_{1} \leq 2 \pi, 0 \leq \phi_{2} \leq 2 \pi, 0 \leq \theta \leq \pi / 2$, as

$$
\begin{aligned}
& d S=P\left[\frac{1}{2} T_{\phi_{1} \phi_{1}} \sigma_{11}^{2}+T_{\phi_{1} \phi_{2}} \sigma_{11} \sigma_{21}+T_{\phi_{1} \theta} \sigma_{11} \sigma_{31}+\frac{1}{2} T_{\phi_{2} \phi_{2}} \sigma_{21}^{2}+T_{\phi_{2} \theta} \sigma_{21} \sigma_{31}\right. \\
&+\frac{1}{2} T_{\theta \theta} \sigma_{31}^{2}+\left(m_{1}+\sigma_{11} \sigma_{41}\right) T_{\phi_{1}}+\left(m_{2}+\right.\left.\left.\sigma_{21} \sigma_{41}\right) T_{\phi_{2}}+\left(m_{3}+\sigma_{31} \sigma_{41}\right) T_{\theta}+m_{4} T\right] d t \\
&+P\left(T_{\phi_{1}} \sigma_{11}+T_{\phi_{2}} \sigma_{21}+T_{\theta} \sigma_{31}+T \sigma_{41}\right) d W(t) .
\end{aligned}
$$

If the transformation function $T\left(\phi_{1}, \phi_{2}, \theta\right)$ is bounded and nonsingular, both processes $P$ and $S$ possess the same stability behavior. Therefore, $T\left(\phi_{1}, \phi_{2}, \theta\right)$ is chosen so that the drift term, of the Ito differential (9), does not depend on the phase processes $\phi_{1}, \phi_{2}$, and $\theta$, so that

$$
d S=\Lambda(p) S d t+S T^{-1}\left(T_{\phi_{1}} \sigma_{11}+T_{\phi_{2}} \sigma_{21}+T_{\theta} \sigma_{31}+T \sigma_{41}\right) d W(t) .
$$

By comparing (9) and (10), we see that such a $T\left(\phi_{1}, \phi_{2}, \theta\right)$ is given by the following equation:

$$
\begin{aligned}
\frac{1}{2} T_{\phi_{1} \phi_{1}} \sigma_{11}^{2}+T_{\phi_{1} \phi_{2}} \sigma_{11} \sigma_{21}+T_{\phi_{1} \theta} \sigma_{11} \sigma_{31}+\frac{1}{2} T_{\phi_{2} \phi_{2}} \sigma_{21}^{2}+T_{\phi_{2} \theta} \sigma_{21} \sigma_{31}+\frac{1}{2} T_{\theta \theta} \sigma_{31}^{2} \\
+\left(m_{1}+\sigma_{11} \sigma_{41}\right) T_{\phi_{1}}+\left(m_{2}+\sigma_{21} \sigma_{41}\right) T_{\phi_{2}}+\left(m_{3}+\sigma_{31} \sigma_{41}\right) T_{\theta}+m_{4} T=\Lambda(p) T .
\end{aligned}
$$

To avoid lengthy calculations, the analysis presented in this section considers the special case where $k_{11}=k_{22}=0$, so that

$$
\left[L_{1}+\varepsilon L_{2}\right] T\left(\phi_{1}, \phi_{2}, \theta\right)=\Lambda(p) T\left(\phi_{1}, \phi_{2}, \theta\right) .
$$

Here $L_{1}$ and $L_{2}$ are the following first- and second-order differential operators (again we suppress the dependence of the coefficients on $\left.\phi_{1}, \phi_{2}, \theta\right)$ :

$$
\begin{aligned}
& L_{1}=\omega_{1} \frac{\partial}{\partial \phi_{1}}+\omega_{2} \frac{\partial}{\partial \phi_{2}}, \\
& L_{2}=a_{1} \frac{\partial^{2}}{\partial \phi_{1}^{2}}+a_{2} \frac{\partial^{2}}{\partial \phi_{1} \partial \phi_{2}}+a_{3} \frac{\partial^{2}}{\partial \phi_{1} \partial \theta}+a_{4} \frac{\partial^{2}}{\partial \phi_{2}^{2}}+a_{5} \frac{\partial^{2}}{\partial \phi_{2} \partial \theta}+a_{6} \frac{\partial^{2}}{\partial \theta^{2}}+b_{1} \frac{\partial}{\partial \phi_{1}}+b_{2} \frac{\partial}{\partial \phi_{2}}+b_{3} \frac{\partial}{\partial \theta}+c,
\end{aligned}
$$


where

$a_{1}=\frac{k_{12}^{2} \sigma^{2}}{4 \omega_{1}^{2}} \cos ^{2} \phi_{1} \cos ^{2} \phi_{2} \tan ^{2} \theta, \quad a_{2}=\frac{k_{12} k_{21} \sigma^{2}}{2 \omega_{1} \omega_{2}} \cos ^{2} \phi_{1} \cos ^{2} \phi_{2}$,
$a_{3}=\frac{\sigma^{2}}{4}\left(-\frac{k_{12}^{2}}{\omega_{1}^{2}} \sin 2 \phi_{1} \cos ^{2} \phi_{2} \sin ^{2} \theta+\frac{k_{12} k_{21}}{\omega_{1} \omega_{2}} \sin 2 \phi_{2} \cos ^{2} \phi_{1} \cos ^{2} \theta\right) \tan \theta$,

$a_{4}=\frac{k_{21}^{2} \sigma^{2}}{4 \omega_{2}^{2}} \cos ^{2} \phi_{1} \cos ^{2} \phi_{2} \cot ^{2} \theta, \quad a_{6}=\frac{\sigma^{2}}{4}\left(-\frac{k_{12}}{\omega_{1}} \sin \phi_{1} \cos \phi_{2} \sin ^{2} \theta+\frac{k_{21}}{\omega_{2}} \sin \phi_{2} \cos \phi_{1} \cos ^{2} \theta\right)^{2}$,

$a_{5}=\frac{\sigma^{2}}{4}\left(-\frac{k_{12} k_{21}}{\omega_{1} \omega_{2}} \sin 2 \phi_{1} \cos ^{2} \phi_{2} \sin ^{2} \theta+\frac{k_{21}^{2}}{\omega_{2}^{2}} \sin 2 \phi_{2} \cos ^{2} \phi_{1} \cos ^{2} \theta\right) \cot \theta$,

$b_{1}=-\left(\zeta_{1} \omega_{1}+\frac{k_{12}^{2} \sigma^{2}}{4 \omega_{1}^{2}}\left(\tan ^{2} \theta-p \sin ^{2} \theta\right) \cos ^{2} \phi_{2}\right) \sin 2 \phi_{1}+\frac{k_{12} k_{21} \sigma^{2}}{4 \omega_{1} \omega_{2}} p \cos ^{2} \phi_{1} \sin ^{2} \theta \sin 2 \phi_{2}$,

$b_{2}=-\left(\zeta_{2} \omega_{2}+\frac{k_{21}^{2} \sigma^{2}}{4 \omega_{2}^{2}}\left(\cot ^{2} \theta-p \cos ^{2} \theta\right) \cos ^{2} \phi_{1}\right) \sin 2 \phi_{2}+\frac{k_{12} k_{21} \sigma^{2}}{4 \omega_{1} \omega_{2}} p \cos ^{2} \phi_{2} \cos ^{2} \theta \sin 2 \phi_{1}$,

$b_{3}=\left(\zeta_{1} \omega_{1} \sin ^{2} \phi_{1}-\zeta_{2} \omega_{2} \sin ^{2} \phi_{2}\right) \sin 2 \theta+\frac{\sigma^{2}}{4}\left(\frac{k_{12}^{2}}{\omega_{1}^{2}}\left(-\cos ^{2} \phi_{1} \tan \theta-(p-1) \sin ^{2} \phi_{1} \sin 2 \theta\right) \cos ^{2} \phi_{2} \sin ^{2} \theta\right.$

$\left.+\frac{k_{21}^{2}}{\omega_{2}^{2}}\left(\cos ^{2} \phi_{2} \cot \theta+(p-1) \sin ^{2} \phi_{2} \sin 2 \theta\right) \cos ^{2} \phi_{1} \cos ^{2} \theta+\frac{k_{12} k_{21}}{4 \omega_{1} \omega_{2}}(p-1) \sin 2 \phi_{1} \sin 2 \phi_{2} \cos 2 \theta \sin 2 \theta\right)$,

$c=-2 p\left(\zeta_{1} \omega_{1} \sin ^{2} \phi_{1} \cos ^{2} \theta+\zeta_{2} \omega_{2} \sin ^{2} \phi_{2} \sin ^{2} \theta\right)+\frac{p \sigma^{2}}{4}\left(\frac{k_{12}^{2}}{\omega_{1}^{2}}\left(1+(p-2) \sin ^{2} \phi_{1} \cos ^{2} \theta\right) \cos ^{2} \phi_{2} \sin ^{2} \theta\right.$ $\left.+\frac{k_{21}^{2}}{\omega_{2}^{2}}\left(1+(p-2) \sin ^{2} \phi_{2} \sin ^{2} \theta\right) \cos ^{2} \phi_{1} \cos ^{2} \theta+(p-2) \frac{k_{12} k_{21}}{2 \omega_{1} \omega_{2}} \sin 2 \phi_{1} \sin 2 \phi_{2} \sin ^{2} \theta \cos ^{2} \theta\right)$.

Either (11) or (12) defines a second-order eigenvalue problem for the determination of the unknown transformation function $T\left(\phi_{1}, \phi_{2}, \theta\right)$ and the associated eigenvalue $\Lambda(p)$ or Lyapunov exponent of the $p$-th mean. From (10) the eigenvalue $\Lambda(p)$ is seen to be the $p$-th moment Lyapunov exponent of the system (4). This approach was first applied by Wedig [1988] to derive the eigenvalue problem for the moment Lyapunov exponent of a 2D linear Itô stochastic system. In the following section, the method of regular perturbation is applied to the eigenvalue problem (11) to obtain a weak noise expansion of the moment Lyapunov exponent for the system (4).

\section{Weak noise expansion of the moment Lyapunov exponent}

Applying the method of regular perturbation, both the moment Lyapunov exponent $\Lambda(p)$ and the eigenfunction $T\left(\phi_{1}, \phi_{2}, \theta\right)$ are expanded in power series of $\varepsilon$ as

$$
\begin{aligned}
\Lambda(p) & =\Lambda_{0}(p)+\varepsilon \Lambda_{1}(p)+\varepsilon^{2} \Lambda_{2}(p)+\ldots+\varepsilon^{n} \Lambda_{n}(p)+\ldots \\
T\left(\phi_{1}, \phi_{2}, \theta\right) & =T_{0}\left(\phi_{1}, \phi_{2}, \theta\right)+\varepsilon T_{1}\left(\phi_{1}, \phi_{2}, \theta\right)+\varepsilon^{2} T_{2}\left(\phi_{1}, \phi_{2}, \theta\right)+\ldots+\varepsilon^{n} T_{n}\left(\phi_{1}, \phi_{2}, \theta\right)+\ldots
\end{aligned}
$$


Substituting the perturbation series (13) into the eigenvalue problem (12) and equating terms with equal powers of $\varepsilon$ leads to the following equations:

$$
\begin{aligned}
\varepsilon^{0}: L_{1} T_{0}\left(\phi_{1}, \phi_{2}, \theta\right) & =\Lambda_{0}(p) T_{0}\left(\phi_{1}, \phi_{2}, \theta\right) \\
\varepsilon^{1}: L_{1} T_{1}\left(\phi_{1}, \phi_{2}, \theta\right)+L_{2} T_{0}\left(\phi_{1}, \phi_{2}, \theta\right) & =\Lambda_{0}(p) T_{1}\left(\phi_{1}, \phi_{2}, \theta\right)+\Lambda_{1}(p) T_{0}\left(\phi_{1}, \phi_{2}, \theta\right), \\
\varepsilon^{2}: L_{1} T_{2}\left(\phi_{1}, \phi_{2}, \theta\right)+L_{2} T_{1}\left(\phi_{1}, \phi_{2}, \theta\right) & =\Lambda_{0}(p) T_{2}\left(\phi_{1}, \phi_{2}, \theta\right) \\
& +\Lambda_{1}(p) T_{1}\left(\phi_{1}, \phi_{2}, \theta\right)+\Lambda_{2}(p) T_{0}\left(\phi_{1}, \phi_{2}, \theta\right), \\
\varepsilon^{3}: L_{1} T_{3}\left(\phi_{1}, \phi_{2}, \theta\right)+L_{2} T_{2}\left(\phi_{1}, \phi_{2}, \theta\right)= & \Lambda_{0}(p) T_{3}\left(\phi_{1}, \phi_{2}, \theta\right)+\Lambda_{1}(p) T_{2}\left(\phi_{1}, \phi_{2}, \theta\right) \\
\vdots & +\Lambda_{2}(p) T_{1}\left(\phi_{1}, \phi_{2}, \theta\right)+\Lambda_{3}(p) T_{0}\left(\phi_{1}, \phi_{2}, \theta\right), \\
\varepsilon^{n}: L_{1} T_{n}\left(\phi_{1}, \phi_{2}, \theta\right)+L_{2} T_{n-1}\left(\phi_{1}, \phi_{2}, \theta\right)= & \Lambda_{0}(p) T_{n}\left(\phi_{1}, \phi_{2}, \theta\right)+\Lambda_{1}(p) T_{n-1}\left(\phi_{1}, \phi_{2}, \theta\right) \\
& +\ldots+\Lambda_{n}(p) T_{0}\left(\phi_{1}, \phi_{2}, \theta\right),
\end{aligned}
$$

where each function $T_{i}\left(\phi_{1}, \phi_{2}, \theta\right), i=0,1,2, \ldots$, must be positive and periodic in the range $0 \leq \phi_{1} \leq 2 \pi$, $0 \leq \phi_{2} \leq 2 \pi, 0 \leq \theta \leq \pi / 2$.

3.1. Zeroth-order perturbation. The zeroth-order perturbation equation is $L_{1} T_{0}=\Lambda_{0}(p) T_{0}$ or

$$
\omega_{1} \frac{\partial T_{0}\left(\phi_{1}, \phi_{2}, \theta\right)}{d \phi_{1}}+\omega_{2} \frac{\partial T_{0}\left(\phi_{1}, \phi_{2}, \theta\right)}{d \phi_{2}}=\Lambda_{0}(p) T_{0}\left(\phi_{1}, \phi_{2}, \theta\right) .
$$

From the properties of the moment Lyapunov exponent, it is known that

$$
\Lambda(0)=\Lambda_{0}(0)+\varepsilon \Lambda_{1}(0)+\varepsilon^{2} \Lambda_{2}(0)+\cdots+\varepsilon^{n} \Lambda_{n}(0)=0,
$$

which results in $\Lambda_{n}(0)=0$ for $n=0,1,2,3, \ldots$ Since the eigenvalue problem (15) does not contain $p$, the eigenvalue $\Lambda_{0}(p)$ is independent of $p$. Hence, $\Lambda_{0}(0)=0$ leads to $\Lambda_{0}(p)=0$. Then (15) has a periodic solution if and only if

$$
\Lambda_{0}(p)=0, \quad T_{0}\left(\phi_{1}, \phi_{2}, \theta\right)=1 .
$$

3.2. First-order perturbation. The first-order perturbation equation is

$$
L_{1} T_{1}\left(\phi_{1}, \phi_{2}, \theta\right)+L_{2} T_{0}\left(\phi_{1}, \phi_{2}, \theta\right)=\Lambda_{0}(p) T_{1}\left(\phi_{1}, \phi_{2}, \theta\right)+\Lambda_{1}(p) T_{0}\left(\phi_{1}, \phi_{2}, \theta\right) .
$$

This has a periodic solution if and only if

$$
\int_{0}^{2 \pi} \int_{0}^{2 \pi} \int_{0}^{\pi / 2}\left[L_{2} \cdot 1-\Lambda_{1}(p)\right] d \phi_{1} d \phi_{2} d \theta=0
$$

so we have

$$
\begin{aligned}
\Lambda_{1}(p) & =\frac{1}{2 \pi^{3}} \int_{0}^{2 \pi} \int_{0}^{2 \pi} \int_{0}^{\pi / 2} c\left(\phi_{1}, \phi_{2}, \theta\right) d \phi_{1} d \phi_{2} d \theta \\
& =p\left[\frac{3 \sigma^{2}}{64}\left(\frac{k_{12}^{2}}{\omega_{1}^{2}}+\frac{k_{21}^{2}}{\omega_{2}^{2}}\right)-\frac{1}{2}\left(\zeta_{1} \omega_{1}+\zeta_{2} \omega_{2}\right)\right]+p^{2} \frac{\sigma^{2}}{128}\left(\frac{k_{12}^{2}}{\omega_{1}^{2}}+\frac{k_{21}^{2}}{\omega_{2}^{2}}\right) .
\end{aligned}
$$


Now the first-order perturbation equation reduces to

$$
\omega_{1} \frac{\partial T_{1}\left(\phi_{1}, \phi_{2}, \theta\right)}{d \phi_{1}}+\omega_{2} \frac{\partial T_{1}\left(\phi_{1}, \phi_{2}, \theta\right)}{d \phi_{2}}+c\left(\phi_{1}, \phi_{2}, \theta\right)=\Lambda_{1}(p)
$$

Since it is assumed that the frequencies $\omega_{1}$ and $\omega_{2}$ are not commensurable, the general periodic solution (21) cannot be obtained explicitly for the eigenfunction $T_{1}\left(\phi_{1}, \phi_{2}, \theta\right)$. Therefore it is possible to obtain the moment Lyapunov exponent only in the first-order perturbation.

3.3. Moment Lyapunov exponent, Lyapunov exponent, and stability conditions. We next obtain the weak noise expansion of the moment Lyapunov exponent in the first-order perturbation for the system (4):

$$
\begin{aligned}
\Lambda(p) & =\varepsilon \Lambda_{1}(p)+O\left(\varepsilon^{2}\right) \\
& =\varepsilon p\left[\frac{3 \sigma^{2}}{64}\left(\frac{k_{12}^{2}}{\omega_{1}^{2}}+\frac{k_{21}^{2}}{\omega_{2}^{2}}\right)-\frac{1}{2}\left(\zeta_{1} \omega_{1}+\zeta_{2} \omega_{2}\right)\right]+\varepsilon p^{2} \frac{\sigma^{2}}{128}\left(\frac{k_{12}^{2}}{\omega_{1}^{2}}+\frac{k_{21}^{2}}{\omega_{2}^{2}}\right)+O\left(\varepsilon^{2}\right) .
\end{aligned}
$$

The Lyapunov exponent for system (4) can be obtained from (22) by using a property of the moment Lyapunov exponent

$$
\lambda=\left.\frac{d \Lambda(p)}{d p}\right|_{p=0}=\varepsilon \lambda_{1}+O\left(\varepsilon^{2}\right)=\varepsilon\left[\frac{3 \sigma^{2}}{64}\left(\frac{k_{12}^{2}}{\omega_{1}^{2}}+\frac{k_{21}^{2}}{\omega_{2}^{2}}\right)-\frac{1}{2}\left(\zeta_{1} \omega_{1}+\zeta_{2} \omega_{2}\right)\right]+O\left(\varepsilon^{2}\right) .
$$

Using the result above for the moment Lyapunov exponent, with the definition of the moment stability $\Lambda_{q(t)}<0$, we determine analytically the $p$-th moment stability boundary in the first-order perturbation for various values of $p=1,2,4$, respectively, as

$\zeta_{1} \omega_{1}+\zeta_{2} \omega_{2}>\frac{7 \sigma^{2}}{64}\left(\frac{k_{12}^{2}}{\omega_{1}^{2}}+\frac{k_{21}^{2}}{\omega_{2}^{2}}\right), \quad \zeta_{1} \omega_{1}+\zeta_{2} \omega_{2}>\frac{\sigma^{2}}{8}\left(\frac{k_{12}^{2}}{\omega_{1}^{2}}+\frac{k_{21}^{2}}{\omega_{2}^{2}}\right), \quad \zeta_{1} \omega_{1}+\zeta_{2} \omega_{2}>\frac{5 \sigma^{2}}{32}\left(\frac{k_{12}^{2}}{\omega_{1}^{2}}+\frac{k_{21}^{2}}{\omega_{2}^{2}}\right)$.

It is known that the system is asymptotically stable only if the Lyapunov exponent $\lambda_{q(t)}$ is negative. Then, (32) is employed to determine the almost-sure stability boundary of the system (4)

$$
\zeta_{1} \omega_{1}+\zeta_{2} \omega_{2}>\frac{3 \sigma^{2}}{32}\left(\frac{k_{12}^{2}}{\omega_{1}^{2}}+\frac{k_{21}^{2}}{\omega_{2}^{2}}\right)
$$

\section{Application to a beam under stochastic load}

The results obtained in the previous section in the context of real engineering applications show how these results can be applied to physical problems. To this end, we consider the flexural torsional instability of a thin rectangular beam of length $L$ subjected to a stochastically fluctuating follower force (Figure 1). It is assumed that the simply supported beam is bent by a stochastically varying central load $P(t)$. The 


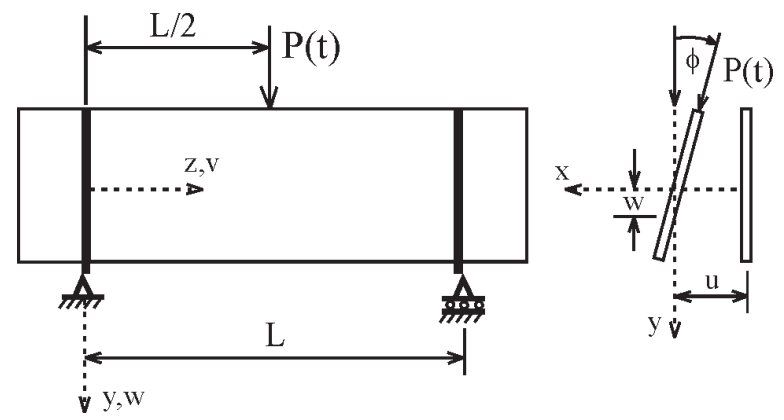

Figure 1. Thin rectangular beam subjected to stochastic excitation.

motion of the beam is governed by the partial differential equations [Bolotin 1964]

$$
\begin{aligned}
m \frac{\partial^{2} w}{\partial t^{2}}+d_{w} \frac{\partial w}{\partial t}+E I_{x} \frac{\partial^{4} w}{\partial z^{4}} & =0, \\
m \frac{\partial^{2} u}{\partial t^{2}}+d_{u} \frac{\partial u}{\partial t}+E I_{y} \frac{\partial^{4} u}{\partial z^{4}}+\frac{\partial^{2}(M(z, t) \phi)}{\partial z^{2}}+P(t) \delta\left(\frac{L}{2}-z\right) \phi & =0, \\
m \rho^{2} \frac{\partial^{2} \phi}{\partial t^{2}}+d_{\phi} \frac{\partial \phi}{\partial t}-G J \frac{\partial^{4} \phi}{\partial z^{4}}+M(z, t) \frac{\partial^{2} u}{\partial z^{2}} & =0,
\end{aligned}
$$

where $u(z, t)$ and $w(z, t)$ denote the $x$ and $y$ components of the deflection of the beam center line and $\phi$ is the angle of twist of the cross-section. The quantities $E I_{x}, E I_{y}$, and $G J$ are the flexural and torsional rigidities of the cross-section and $d_{w}, d_{u}$, and $d_{\phi}$ are the viscous damping coefficients. In addition, $m$ denotes the mass per unit length, and $\rho$ is the polar radius of gyration of the cross-section. The function $M(z, t)$ can be expressed in terms of the applied stochastic load as

$$
M(z, t)= \begin{cases}\frac{1}{2} P(t) \cdot z, & 0 \leq z \leq \frac{1}{2} L \\ \frac{1}{2} P(t) \cdot(L-z), & \frac{1}{2} L \leq z \leq L .\end{cases}
$$

It is obvious that (25) is uncoupled from the other two equations and describes the ordinary random vibration of the beam in the plane of its largest rigidity with the inhomogeneous boundary conditions given by

$$
E I_{x} \frac{\partial^{2} w}{\partial z^{2}}(0, t)=-M(0, t)=E I_{x} \frac{\partial^{2} w}{\partial z^{2}}(L, t)=-M(L, t) .
$$

The other two equations form a pair of coupled partial differential equations with stochastic coefficients subjected to homogeneous boundary conditions given by

$$
u(0, t)=u(L, t)=\frac{\partial^{2} u}{\partial z^{2}}(0, t)=\frac{\partial^{2} u}{\partial z^{2}}(L, t), \quad \phi(0, t)=\phi(L, t)=0 .
$$

Consider the shape function $\sin (\pi z / L)$, which satisfies the boundary conditions for the first mode vibration. The displacement $u(z, t)$ and twist $\phi(z, t)$ can be described by

$$
u(z, t)=\rho q_{1}(t) \sin \left(\frac{\pi z}{L}\right), \quad \phi(z, t)=q_{2}(t) \sin \left(\frac{\pi z}{L}\right) .
$$


Substituting these into the partial differential equations (26) and (27) yields

$$
\begin{aligned}
& \ddot{q}_{1}+\omega_{1}^{2} q_{1}+2 \varepsilon \zeta_{1} \omega_{1} \dot{q}_{1}+\sqrt{\varepsilon} k_{12} q_{2} \xi(t)=0, \\
& \ddot{q}_{2}+\omega_{2}^{2} q_{2}+2 \varepsilon \zeta_{2} \omega_{2} \dot{q}_{2}+\sqrt{\varepsilon} k_{21} q_{1} \xi(t)=0
\end{aligned}
$$

where

$$
\begin{gathered}
\omega_{1}^{2}=\frac{\pi^{4}}{m L^{4}} E I_{y}, \quad \omega_{2}^{2}=\frac{\pi^{2}}{m \rho^{2} L^{2}} G J, \quad 2 \varepsilon \zeta_{1} \omega_{1}=\frac{d_{u}}{m}, \quad 2 \varepsilon \zeta_{2} \omega_{2}=\frac{d_{\phi}}{\rho^{2} m}, \\
k_{12}=\frac{1}{2 m L \rho}\left(7-\frac{\pi^{2}}{4}\right), \quad k_{12}=-\frac{1}{2 m L \rho}\left(1+\frac{\pi^{2}}{4}\right), \quad \xi(t)=\frac{P(t)}{P_{c r}}, \quad P_{c r}=\frac{4\left|\omega_{1}^{2}-\omega_{2}^{2}\right| m L \rho}{\sqrt{\left(28-\pi^{2}\right)\left(4+\pi^{2}\right)}}
\end{gathered}
$$

Here, $\xi(t)$ is assumed to be a stationary stochastic process.

\section{Numerical determination of the $p$-th moment Lyapunov exponent and conclusions}

The numerical determination of the $p$-th moment Lyapunov exponents is important in assessing the validity and the ranges of applicability of the approximate analytical results. For systems of large dimensions, it is very difficult, if is not impossible, to obtain analytical results. In many engineering applications, the amplitude of noise excitation is not small and approximate analytical methods, such as the method of perturbation or the method of stochastic averaging, cannot be applied. Therefore, numerical approaches have to be employed to evaluate the moment Lyapunov exponents. The numerical approach is based on expanding the exact solution of the system of Itô stochastic differential equations, (33), in powers of the time increment $h$ and the small parameter $\varepsilon$, as proposed in [Milstein and Tret'yakov 1997]. The state vector of the system (4) is to be rewritten as a system of Itô stochastic differential equations with low noise in the form

$$
\begin{aligned}
d x_{1} & =\omega_{1} x_{2} d t=\left[\tilde{a}_{1}(t, \boldsymbol{X})+\varepsilon \tilde{b}_{1}(t, \boldsymbol{X})\right] d t+\sqrt{\varepsilon} \sigma_{11}(t, \boldsymbol{X}) d w(t), \\
d x_{2} & =-\omega_{1}\left(x_{1}+2 \varepsilon \zeta_{1} x_{2}\right) d t-\sqrt{\varepsilon} \sigma\left(\frac{k_{11}}{\omega_{1}} x_{1}+\frac{k_{12}}{\omega_{1}} x_{3}\right) d w(t) \\
& =\left[\tilde{a}_{2}(t, \boldsymbol{X})+\varepsilon \tilde{b}_{2}(t, \boldsymbol{X})\right] d t+\sqrt{\varepsilon} \sigma_{21}(t, \boldsymbol{X}) d w(t), \\
d x_{3} & =\omega_{2} x_{4} d t=\left[\tilde{a}_{3}(t, \boldsymbol{X})+\varepsilon \tilde{b}_{3}(t, \boldsymbol{X})\right] d t+\sqrt{\varepsilon} \sigma_{31}(t, \boldsymbol{X}) d w(t), \\
d x_{4} & =-\omega_{2}\left(x_{3}+2 \varepsilon \zeta_{2} x_{4}\right) d t-\sqrt{\varepsilon} \sigma\left(\frac{k_{21}}{\omega_{2}} x_{1}+\frac{k_{22}}{\omega_{2}} x_{3}\right) d w(t) \\
& =\left[\tilde{a}_{4}(t, \boldsymbol{X})+\varepsilon \tilde{b}_{4}(t, \boldsymbol{X})\right] d t+\sqrt{\varepsilon} \sigma_{41}(t, \boldsymbol{X}) d w(t) .
\end{aligned}
$$

For numerical solutions of the stochastic differential (33) the weak Runge-Kutta method with error $R=O\left(h^{4}+\varepsilon^{4} h\right)$ may be applied to evaluate numerically the $p$-th moment $E\left[\|\boldsymbol{X}\|^{p}\right]$. A total of $N$ samples of the solutions of (33) are generated. The weak Runge-Kutta scheme of the $s$-th realization of (33) at the $(k+1)$-th iteration with $t^{(k+1)}-t^{(k)}=h$, where $h$ is the time step of integration, is given by 
[Milstein and Tret'yakov 1997]:

$$
\begin{gathered}
X_{j}^{(k+1)}=X_{j}^{(k)}+\frac{\varepsilon^{1 / 2} h^{1 / 2}}{6}\left[\sigma_{j 1}\left(t^{(k)}, \boldsymbol{X}^{(k)}\right)\left(\xi^{(k)}+6 \eta^{(k)}\right)+4 \sigma_{j 1}\left(t^{(k+1 / 2)}, \boldsymbol{X}^{(k)}+\frac{\boldsymbol{K}_{2}}{2}\right) \cdot \xi^{(k)}\right. \\
\left.+\sigma_{j 1}\left(t^{(k+1)}, \boldsymbol{X}^{(k)}+\boldsymbol{K}_{1}\right) \cdot\left(\xi^{(k)}-6 \eta^{(k)}\right)\right] \\
+\frac{h}{2}\left[\tilde{a}_{j}\left(t^{(k)}, \boldsymbol{X}^{(k)}+\varepsilon^{1 / 2} h^{1 / 2} \sigma_{j 1}\left(t^{(k)}, \boldsymbol{X}^{(k)}\right) \eta^{(k)}\right)-\tilde{a}_{j}\left(t^{(k)}, \boldsymbol{X}^{(k)}-\varepsilon^{1 / 2} h^{1 / 2} \sigma_{j 1}\left(t^{(k)}, \boldsymbol{X}^{(k)}\right) \eta^{(k)}\right)\right] \\
+\frac{1}{6}\left(\boldsymbol{K}_{1}+2 \boldsymbol{K}_{2}+2 \boldsymbol{K}_{3}+\boldsymbol{K}_{4}\right)+\frac{\varepsilon}{4}\left(\boldsymbol{l}_{1}+3 \boldsymbol{l}_{2}\right), \quad j=1,2,3,4, \quad
\end{gathered}
$$

where

$$
\begin{aligned}
\boldsymbol{\sigma}_{1} & =\left(\sigma_{11}(t, \boldsymbol{X}), \sigma_{21}(t, \boldsymbol{X}), \sigma_{31}(t, \boldsymbol{X}), \sigma_{41}(t, \boldsymbol{X})\right), \\
\boldsymbol{X} & =\left(x_{1}, x_{2}, x_{3}, x_{4}\right), \\
\boldsymbol{a} & =\left(\tilde{a}_{1}(t, \boldsymbol{X}), \tilde{a}_{2}(t, \boldsymbol{X}), \tilde{a}_{3}(t, \boldsymbol{X}), \tilde{a}_{4}(t, \boldsymbol{X})\right), \\
\boldsymbol{b} & =\left(\tilde{b}_{1}(t, \boldsymbol{X}), \tilde{b}_{2}(t, \boldsymbol{X}), \tilde{b}_{3}(t, \boldsymbol{X}), \tilde{b}_{4}(t, \boldsymbol{X})\right), \\
\boldsymbol{K}_{1} & =\left(K_{11}, K_{12}, K_{13}, K_{14}\right)=h \cdot \tilde{\boldsymbol{a}}\left(t^{(k)}, \boldsymbol{X}^{(k)}\right), \\
\boldsymbol{K}_{2} & =\left(K_{21}, K_{22}, K_{23}, K_{24}\right)=h \cdot \tilde{\boldsymbol{a}}\left(t^{(k+1 / 2)}, \boldsymbol{X}^{(k)}+\frac{\boldsymbol{K}_{1}}{2}\right), \\
\boldsymbol{K}_{3} & =\left(K_{31}, K_{32}, K_{33}, K_{34}\right)=h \cdot \tilde{\boldsymbol{a}}\left(t^{(k+1 / 2)}, \boldsymbol{X}^{(k)}+\varepsilon^{1 / 2} h^{1 / 2} \sigma_{1}\left(t^{(k)}, \boldsymbol{X}^{(k)}\right) \xi^{(k)}+\frac{\boldsymbol{K}_{2}}{2}+\varepsilon \frac{\boldsymbol{l}_{1}}{4}+\varepsilon \frac{3}{4} \boldsymbol{l}_{2}\right), \\
\boldsymbol{K}_{4} & =\left(K_{41}, K_{42}, K_{43}, K_{44}\right)=h \cdot \tilde{\boldsymbol{a}}\left(t^{(k+1)}, \boldsymbol{X}^{(k)}+\varepsilon^{1 / 2} h^{1 / 2} \boldsymbol{\sigma}_{1}\left(t^{(k+1)}, \boldsymbol{X}^{(k)}+\boldsymbol{K}_{1}\right) \xi^{(k)}+\boldsymbol{K}_{3}+\varepsilon \boldsymbol{l}_{1}\right), \\
\boldsymbol{l}_{1} & =\left(l_{11}, l_{12}, l_{13}, l_{14}\right)=h \cdot \tilde{\boldsymbol{b}}\left(t^{(k)}, \boldsymbol{X}^{(k)}\right), \\
\boldsymbol{l}_{2} & =\left(l_{21}, l_{22}, l_{23}, l_{24}\right)=h \cdot \tilde{\boldsymbol{b}}\left(t^{(k+2 / 3)}, \boldsymbol{X}^{(k)}+\frac{2}{9} \boldsymbol{K}_{1}+\frac{4}{9} \boldsymbol{K}_{2}\right),
\end{aligned}
$$

and $\xi$ and $\eta$ are random variables simulated using the two-point distribution

$$
P(\xi=-1)=P(\xi=1)=\frac{1}{2}, \quad P\left(\eta=-\frac{1}{\sqrt{12}}\right)=P\left(\eta=\frac{1}{\sqrt{12}}\right)=\frac{1}{2} .
$$

Having obtained $N$ samples of the solutions of the stochastic differential equations (33) the $p$-th moment can be determined as follows:

$$
E\left[\left\|\boldsymbol{X}^{(k+1)}\right\|^{p}\right]=\frac{1}{N} \sum_{s=1}^{N}\left\|\boldsymbol{X}_{s}^{(k+1)}\right\|^{p}, \quad\left\|\boldsymbol{X}_{s}^{(k+1)}\right\|=\sqrt{\left(\boldsymbol{X}_{s}^{(k+1)}\right)^{T}\left(\boldsymbol{X}_{s}^{(k+1)}\right)} .
$$

By the Monte Carlo technique we numerically calculate the $p$-th moment Lyapunov exponent for all the values of $p$ of interest, defined as

$$
\Lambda(p)=\frac{1}{T} \ln E\left[\left\|\boldsymbol{X}_{(T)}\right\|^{p}\right] .
$$

The function $\Lambda(p)$ in the limit of large time $(T \rightarrow \infty)$ tends to the moment Lyapunov exponent $\Lambda(p)$. In this paper, a singular perturbation method is applied to obtain first-order low noise expansions of the moment Lyapunov exponent of a coupled white noise driven, two-degree-of-freedom system. The 


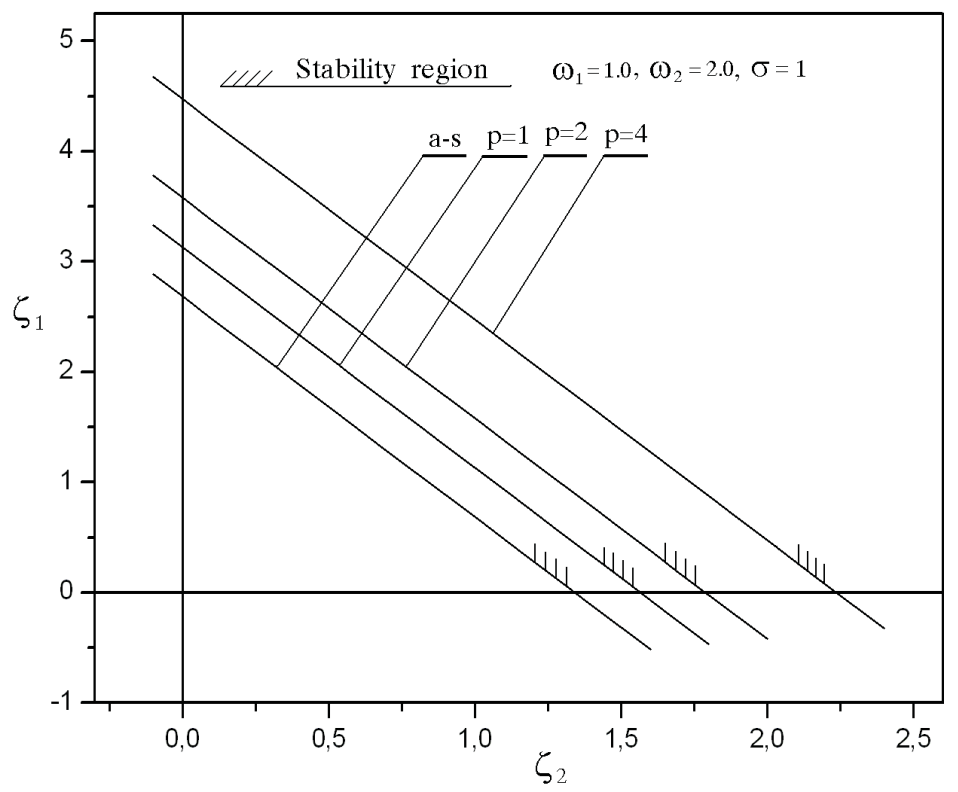

Figure 2. Stability regions for almost-sure (a-s) and $p$-th moment stability for $k_{12}=5$, $k_{21}=-3.82$.

Lyapunov exponent is determined by using the relationship between the moment Lyapunov exponent and the Lyapunov exponent. The slope of the moment Lyapunov exponent curve at $p=0$ is the Lyapunov exponent. When the Lyapunov exponent is negative, the system (3) is almost-sure stable with probability 1 ; otherwise it is unstable. The results obtained above can be directly applied to analyze the flexural-torsional stability of a thin rectangular elastic beam of length $L$ subjected to a stochastic follower force. For the purpose of illustration, in the numerical study we consider set system parameters $k_{12}=5$, $k_{21}=-3.82, \omega_{1}=1, \omega_{2}=2$, and $\sigma=1$. Figure 2 shows the almost-sure and $p$-th moment stability boundaries with respect to the damping coefficients $\zeta_{1}$ and $\zeta_{2}$. Note that the moment stability boundaries are more conservative than the almost-sure boundary. These boundaries become increasingly conservative as $p$ increases. Numerical determination of the $p$-th moment Lyapunov exponent is important in assessing the validity and the range of applicability of the approximate analytical results obtained for stochastic systems. The Monte Carlo simulation methods are usually more versatile, especially when the noise excitations cannot be described in a form that can be treated easily using analytical tools. From the central limit theorem, it is well known that the estimated $p$-th moment Lyapunov exponent is a random number, with the mean being the true value of the $p$-th moment Lyapunov exponent and the standard deviation $n_{p} / \sqrt{N}$, where $n_{p}$ is the sample standard deviation determined from the $N$ samples. The standard deviation of the estimated $p$-th moment Lyapunov exponent can be reduced by increasing the number of samples, $N=2000,4000,8000$. The total time of simulation is 5 and $h=0.0005$ is the time step of integration. Numerical results of the $p$-th moment Lyapunov exponent $\Lambda(p)$ from the Monte Carlo simulation, along with Equation (22) (the solid line), are plotted in Figure 3 for $\omega_{1}=1.0, \omega_{2}=2.0$, $\sigma=1, \zeta_{1}=4.5, \zeta_{2}=0.2$ and $\varepsilon=0.025,0.05,0.1$. It is observed that the discrepancies between the approximate analytical results (the solid line) and the numerical results (the dotted line) increase for large values of $\varepsilon$. 

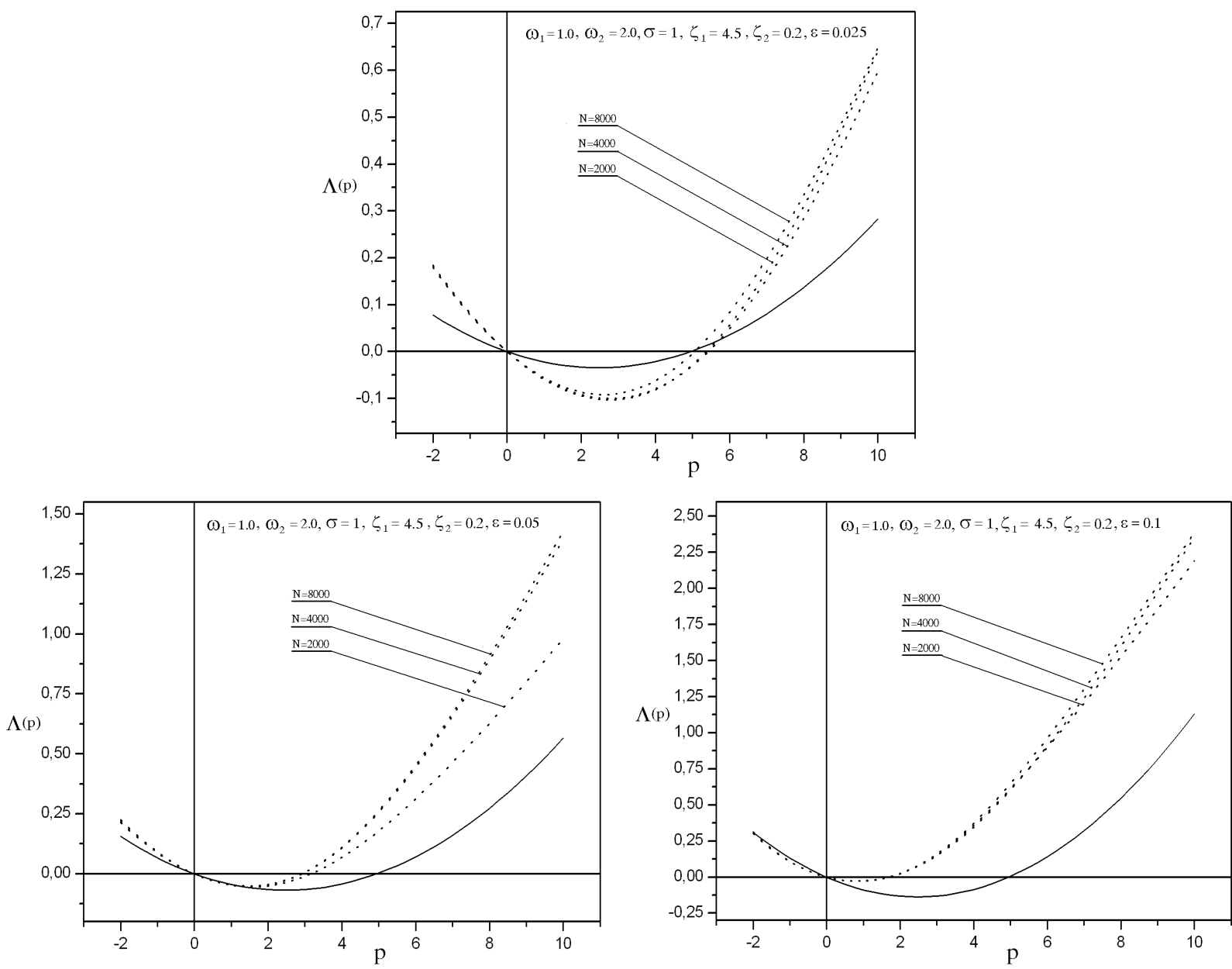

Figure 3. Variation of the moment Lyapunov exponent $\Lambda(p)$ with $p$ for $k_{12}=5, k_{21}=$ -3.82 and different value $\varepsilon$.

\section{References}

[Arnold et al. 1997] L. Arnold, M. M. Doyle, and N. Sri Namachchivaya, "Small noise expansion of moment Lyapunov exponents for two-dimensional systems", Dyn. Stab. Syst. 12:3 (1997), 187-211.

[Bolotin 1964] V. Bolotin, The dynamic stability of elastic systems, Holden-Day, San Francisco, 1964.

[Khasminskii and Moshchuk 1998] R. Khasminskii and N. Moshchuk, "Moment Lyapunov exponent and stability index for linear conservative system with small random perturbation", SIAM J. Appl. Math. 58:1 (1998), 245-256.

[Kozić et al. 2008] P. Kozić, R. Pavlović, and G. Janevski, "Moment Lyapunov exponents of the stochastic parametrical Hill's equation”, Int. J. Solids Struct. 45:24 (2008), 6056-6066.

[Milstein and Tret'yakov 1997] G. N. Milstein and M. V. Tret'yakov, "Numerical methods in the weak sense for stochastic differential equations with small noise", SIAM J. Numer. Anal. 34:6 (1997), 2142-2167.

[Sri Namachchivaya and Van Roessel 2004] N. Sri Namachchivaya and H. J. Van Roessel, "Stochastic stability of coupled oscillators in resonance: a perturbation approach", J. Appl. Mech. (ASME) 71:6 (2004), 759-767.

[Sri Namachchivaya and Vedula 2000] N. Sri Namachchivaya and L. Vedula, "Stabilization of linear systems by noise: application to flow induced oscillations", Dyn. Stab. Syst. 15:2 (2000), 185-208. 
[Sri Namachchivaya et al. 1994] N. Sri Namachchivaya, H. J. Van Roessel, and S. Talwar, "Maximal Lyapunov exponent and almost-sure stability for coupled two-degree-of-freedom stochastic systems", J. Appl. Mech. (ASME) 61:2 (1994), 446-452.

[Wedig 1988] W. Wedig, "Lyapunov exponent of stochastic systems and related bifurcation problem", pp. 315-327 in Stochastic structural dynamics: progress in theory and applications, edited by S. T. Ariaratnam et al., Elsevier, London, 1988.

[Xie 2001] W.-C. Xie, "Moment Lyapunov exponents of a two-dimensional system under real-noise excitation", J. Sound Vib. 239:1 (2001), 139-155.

[Xie 2002] W.-C. Xie, "Moment Lyapunov exponents of a two-dimensional viscoelastic system under bounded noise excitation”, J. Appl. Mech. (ASME) 69:3 (2002), 346-357.

Received 27 Oct 2008. Revised 23 Feb 2009. Accepted 19 Jun 2009.

PREDRAG KozIĆ: kozip@yahoo.com

Department of Mechanical Engineering, University of Niš, Medvedeva 14, 18000 Niš, Serbia

GORAN JANEVSKI: gocky. jane@gmail . com

Department of Mechanical Engineering, University of Niš, Medvedeva 14, 18000 Niš, Serbia

RATKO PAVlović: ratpav@yahoo.com

Department of Mechanical Engineering, University of Niš, Medvedeva 14, 18000 Niš, Serbia 